\title{
Usefulness of a multifunctional snare designed for colorectal hybrid endoscopic submucosal dissection (with video) $\square$
}

\section{(ㅇ) $\odot \ominus$}

\author{
Authors \\ Institutions \\ 1 Department of Gastroenterology, NTT Medical Center \\ Tokyo, Tokyo, Japan \\ 2 Department of Gastroenterology, Omori Red Cross \\ Hospital, Tokyo, Japan \\ submitted 6.7.2017 \\ accepted after revision 14.11.2017 \\ Bibliography \\ DOI https:||doi.org|10.1055/s-0043-124364 | \\ Endoscopy International Open 2018; 06: E249-E253 \\ (c) Georg Thieme Verlag KG Stuttgart · New York \\ ISSN 2364-3722 \\ Corresponding author \\ Ken Ohata, MD, Division of Gastroenterology, NTT Medical \\ Center Tokyo, 5-9-22 Higashi-Gotanda, Shinagawa-ku, \\ Tokyo, 141-8625, Japan \\ Fax: +81-3-34486541 \\ ken.ohata1974@gmail.com
}

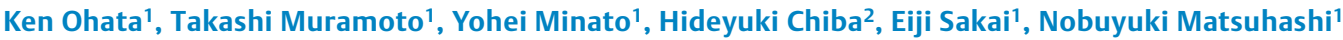

\begin{abstract}
Since colorectal endoscopic submucosal dissection (ESD) remains technically difficult, hybrid ESD was developed as an alternative therapeutic option to achieve en bloc resection of relatively large lesions. In this feasibility study, we evaluated the safety and efficacy of hybrid colorectal ESD using a newly developed multifunctional snare. From June to August 2016, we prospectively enrolled 10 consecutive patients with non-pedunculated intramucosal colorectal tumors $20-30 \mathrm{~mm}$ in diameter. All of the hybrid ESD steps were performed using the "SOUTEN" snare. The knobshaped tip attached to the loop top helps to stabilize the needle-knife, making it less likely to slip during circumferential incision and enables partial submucosal dissection. All of the lesions were curatively resected by hybrid ESD, with a short mean procedure time ( $16.1 \pm 4.8$ minutes). The mean diameters of the resected specimens and tumors were $30.5 \pm 4.9$ and $26.0 \pm 3.5 \mathrm{~mm}$, respectively. No perforations occurred, while delayed bleeding occurred in 1 patient. In conclusion, hybrid ESD using a multifunctional snare enables easy, safe, and cost-effective resection of relatively large colorectal tumors to be achieved. Study registration: UMIN000022545
\end{abstract}

\section{Introduction}

Since endoscopic submucosal dissection (ESD) was initially developed to treat gastric tumors, its application has expanded to the treatment of colorectal tumors. Despite the recent advantages of endoscopic modalities and techniques, colorectal ESD remains technically difficult because of the poor maneuverability of the endoscope, the thin luminal walls, and movements in response to breathing and the cardiac beat, resulting in a high risk of perforation [1]. The difficulty of ESD resides mainly in the process of submucosal dissection, which is strongly affected by the anatomic features of the target lesions. The procedure sometimes fails if the dissection surface cannot be visualized without using adequate traction. Therefore, precise training programs and expert supervision are required to acquire proficiency in performing ESD techniques [2]. Meanwhile, the en bloc resection of tumors $\geq 20 \mathrm{~mm}$ in diameter is considered to be difficult to achieve using endoscopic mucosal resection (EMR).

Hybrid ESD was developed as an alternative therapeutic option to achieve the en bloc resection of relatively large colorectal tumors [3]. Hybrid ESD involves a circumferential incision, partial submucosal dissection, and subsequent snare resection. Because most of the submucosal dissection can be replaced by a snare resection, this technique may decrease the procedure time and the risk of perforation. Recently, a novel multifunctional snare (SOUTEN; Kaneka Medics, Tokyo, Japan) was introduced to enable successful hybrid ESD procedures ( $\triangleright$ Fig.1). Since the knob-shaped tip helps to stabilize the needle-knife, making it less likely to slip during circumferential incision and partial submucosal dissection, all the processes can be completed using 1 device. In this prospective study, we enrolled patients with relatively large, non-pedunculated colorectal tumors and evaluated the usefulness of hybrid ESD using the "SOUTEN" snare for the resection of such lesions. 

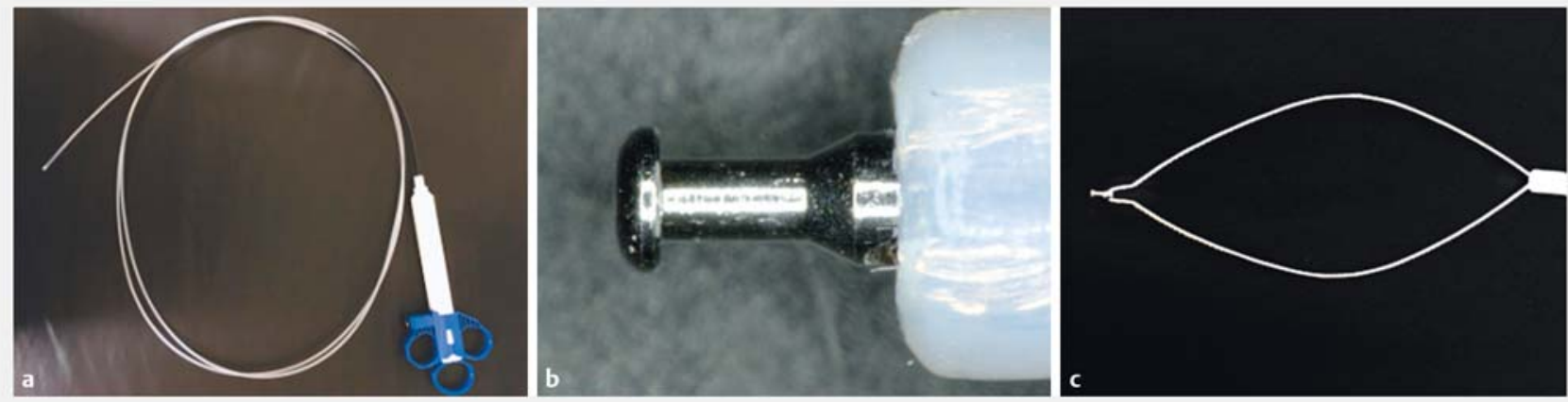

Fig. 1 Details of SOUTEN snare. a A multifunctional snare, designed to achieve hybrid ESD. b A 1.5-mm needle-knife with a knob-shaped tip is attached to the top of the loop.c The length of the loop is $18.5 \mathrm{~mm}$.

\section{Case series}

\section{Patients}

From June to August 2016, the safety and efficacy of hybrid ESD were evaluated for the treatment of non-pedunculated intramucosal colorectal tumors $20-30 \mathrm{~mm}$ in diameter. Among the 22 non-pedunculated colorectal tumors that were larger than $20 \mathrm{~mm}$ in diameter, 10 consecutive patients were prospectively enrolled and treated using hybrid ESD. These lesions are categorized into 2 subtypes based on their macroscopic morphology: laterally spreading tumor, granular type (LST-G) and nongranular type (LST-NG). Lesions involving the ileocecal valve and/or appendiceal orifice were excluded from this study. In addition, lesions with polypoid growth or converging fold and those suspected of exhibiting severe fibrosis were also excluded. The procedure time, the completion rate, R0 and curative resection rate, and the rate of adverse events (e.g. perforation and bleeding) were evaluated. A pathological examination was performed using the Vienna classification [4]. R0 resection was defined as a complete resection with negative lateral and vertical margins when examined pathologically. Curative resection was achieved when both the lateral and vertical margins of the specimen were free of carcinoma and there were no findings of submucosal deep invasion (>1000 $\mu \mathrm{m}$ ) from the muscularis mucosae, lymphatic invasion, vascular involvement, or a poorly differentiated component. Written informed consent was obtained from each patient. This study was approved by the ethics committee of NTT Medical Center Tokyo and was registered with the University Hospital Medical Information Network (UMIN) Clinical Trials as UMIN No. 000022545.

To assess the efficacy and safety of the hybrid ESD, we extracted the clinical data for patients who received conventional ESD randomly from our ESD database performed within the previous year adjusted for tumor size, morphology, and location. The ESD procedures were performed using a dual knife (KD650Q; Olympus Optical Co.). Differences between hybrid and conventional ESD were calculated using the Fisher exact test or an unpaired Student's $t$ test.

\section{Hybrid ESD procedure}

All of the patients received endoscopic treatment under hospitalization. The hybrid ESD procedures were performed using a lower gastrointestinal endoscope with a single channel $3.2 \mathrm{~mm}$ in diameter (PCF-Q260Jl; Olympus Optical Co., Tokyo, Japan) under conscious sedation with flunitrazepam and buprenorphine. Hybrid ESD was performed by 2 endoscopists (HC and TM) who had experience treating more than 100 colorectal ESD cases. Video 1 shows the hybrid ESD procedure using the "SOUTEN" snare for a $30-\mathrm{mm}$ LST-G located in the transverse colon ( $\triangleright$ Fig.2a). After the local injection of sodium hyaluronate solution ( $\boldsymbol{\nabla}$ Fig. $\mathbf{2 b}$ ), a circumferential incision was made using a $1.5-\mathrm{mm}$ needle-knife with a knob-shaped tip without marking ( $\triangleright$ Fig. 2c). The settings of the VIO300 D electrical unit (Erbe Elektromedizin, Tübingen, Germany) were "Endo Cut I" (effect 2, time 2, interval 2) for mucosal incision and "forced coagulation" (effect 2, 45 W) for vessel coagulation

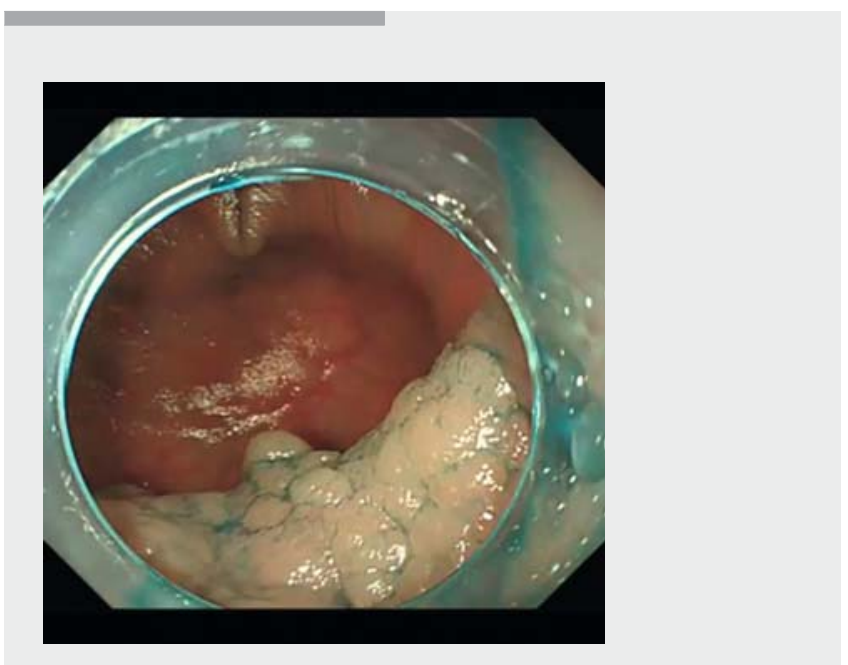

$\checkmark$ Video 1 Hybrid ESD was performed to resect a granular type, laterally spreading tumor (LST-G), $30 \mathrm{~mm}$ in size, located in the transverse colon. A complete resection was achieved within 8 minutes using the "SOUTEN" multifunctional snare, without any adverse events. 

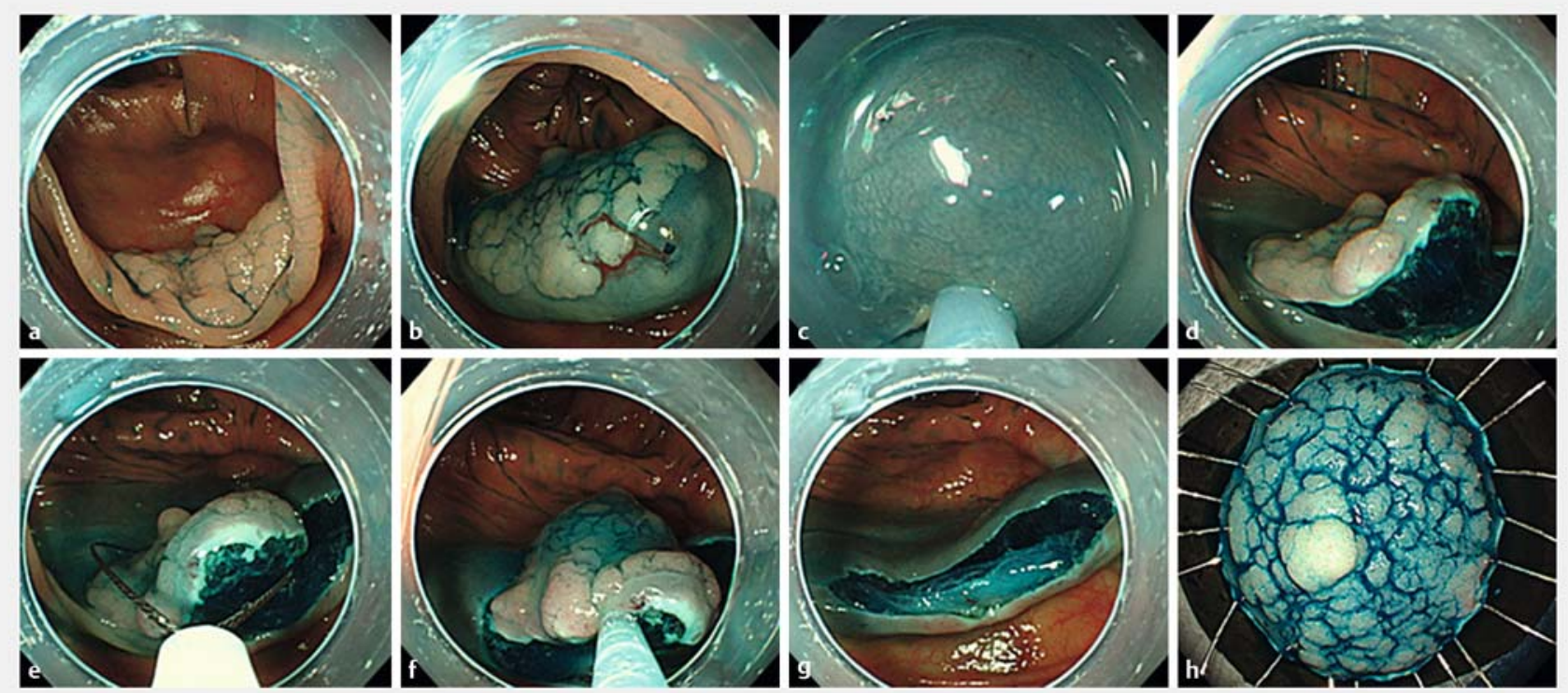

- Fig. 2 Hybrid ESD procedure. a Granular type, laterally spreading tumor (LST-G), $30 \mathrm{~mm}$ in size, located in the transverse colon. b The local injection of sodium hyaluronate solution. $\mathbf{c}$ The mucosal incision was performed using a knob-shaped tip. $\mathbf{d}$ An adequate amount of submucosal dissection was performed. e The snare was placed to fit the dissection plane. $\mathbf{f}$ The tumor was tightly snared. $\mathbf{g}$ Post-hybrid ESD ulcer floor. The lesion was completely resected. $\mathbf{h}$ Resected specimen. An en bloc resection was achieved.

or mucosal dissection/resection. Then, thorough trimming was performed deep enough to cut the muscularis mucosa, especially on the oral side of the lesion. The submucosal dissection was continued until approximately $15 \mathrm{~mm}$ of the submucosa remained undissected ( $\boldsymbol{\nabla}$ Fig. $2 \mathbf{d}$ ). After the snare was placed to fit the dissection plane and was carefully tightened, the lesion was resected ( $\triangleright \mathbf{F i g .} \mathbf{2 e}-\mathbf{h}$ ). If bleeding occurred, hemostasis was also attempted using a knob-shaped tip. The prophylactic closure of the post-procedural ulcer floor was not conducted, otherwise the damage to the muscularis layer was confirmed. A pathological examination of the resected specimen was performed to assess the curability and need for additional surgical resection.

\section{Results}

The clinicopathological characteristics of the resected lesions are shown in $>$ Table 1. The patient age was $57.6 \pm 8.5$ years, and 8 of the 10 patients were male. The prevalence of hypertension, diabetes, cardiovascular disease, and cerebral infarction was $40 \%, 20 \%, 20 \%$, and $10 \%$, respectively. 2 of the 10 patients received anti-platelet therapy.

All of the lesions were successfully resected using the hybrid ESD procedure. The tumor locations were 1, 3, and 6 in the rectum, left-sided colon, and right-sided colon, respectively. The mean procedure time was $16.1 \pm 4.8$ minutes (range, 9-25 minutes). The mean diameter of the resected specimen was $30.5 \pm 4.9 \mathrm{~mm}$ (range, $25-40 \mathrm{~mm}$ ), and the mean tumor size was $26.0 \pm 3.5 \mathrm{~mm}$ (range, $20-32 \mathrm{~mm}$ ). 5 of the 10 lesions were pathologically diagnosed as non-invasive high grade neoplasia (Category 4), while the others were diagnosed as non-in- vasive low grade neoplasia (Category 3). A complete R0 and curative resection was histologically confirmed in all the patients. Delayed bleeding occurred in 1 patient but was controlled by endoscopic hemostasis using conventional clips.

No significant differences in the rates of $\mathrm{R} 0$ resection, curative resection, or adverse events were seen. However, the hybrid ESD group had a significantly shorter procedure time than the conventional ESD group $(16.1 \pm 4.8$ minutes vs. $37.5 \pm 21.1$ minutes, $P>0.001)$. As for medical costs, the cost of an ESD device (KD650Q) is approximately 3 times higher than that of the "SOUTEN" snare.

\section{Discussion}

This was a prospective, open label, single center, consecutive case series evaluating the safety and efficacy of hybrid ESD using a newly developed, multifunctional snare. The rates of en bloc resection with EMR for colorectal tumors larger than $20 \mathrm{~mm}$ are reportedly lower than $50 \%[5,6]$. Recently, Bae et al. conducted a prospective randomized interventional trial and demonstrated that such lesions could be treated safely using the hybrid ESD technique without compromising the en bloc resection rate [7]. As the snare technique is familiar to most endoscopists, it is thought to be easily applied in clinical practice. In the present study, all the lesions were completely resected regardless of the tumor location apart from lesions involving the ileocecal valve and/or appendiceal orifice. Although this was a feasibility study with a small sample size, our results also suggested that hybrid ESD can contribute to the successful en bloc resection of such lesions, similar to conventional ESD. Moreover, the short procedure time and the low risk of intraop- 
- Table 1 Characteristics and therapeutic results for the enrolled patients.

\begin{tabular}{|c|c|c|c|c|c|c|c|c|c|}
\hline Patient & Age & Sex & Tumor location & $\begin{array}{l}\text { Proce- } \\
\text { dure } \\
\text { time, } \\
\text { min }\end{array}$ & $\begin{array}{l}\text { Size of re- } \\
\text { sected spe- } \\
\text { cimen/tu- } \\
\text { mor, mm }\end{array}$ & $\begin{array}{l}\text { Macro- } \\
\text { scopic } \\
\text { classifica- } \\
\text { tion }\end{array}$ & $\begin{array}{l}\text { Patho- } \\
\text { logical } \\
\text { diagnosis }\end{array}$ & $\begin{array}{l}\text { Ro/Cura- } \\
\text { tive re- } \\
\text { section }\end{array}$ & Adverse events \\
\hline 1 & 58 & $\mathrm{~F}$ & Sigmoid colon & 20 & $34 / 27$ & LST-NG & Category 4 & Yes/Yes & None \\
\hline 2 & 41 & M & Cecum & 15 & $32 / 25$ & LST-NG & Category 3 & Yes/Yes & Delayed bleeding \\
\hline 3 & 58 & M & Ascending colon & 19 & $25 / 20$ & LST-NG & Category 3 & Yes/Yes & None \\
\hline 4 & 52 & M & Transverse colon & 8 & $30 / 28$ & LST-G & Category 3 & Yes/Yes & None \\
\hline 5 & 62 & M & Descending colon & 20 & $30 / 30$ & LST-NG & Category 4 & Yes/Yes & None \\
\hline 6 & 68 & M & Rectum & 25 & $40 / 32$ & LST-G & Category 4 & Yes/Yes & None \\
\hline 7 & 70 & M & Ascending colon & 16 & $25 / 24$ & LST-NG & Category 4 & Yes/Yes & None \\
\hline 8 & 54 & M & Cecum & 12 & $35 / 25$ & LST-NG & Category 3 & Yes/Yes & None \\
\hline 9 & 52 & $\mathrm{~F}$ & Sigmoid colon & 15 & $28 / 26$ & LST-NG & Category 4 & Yes/Yes & None \\
\hline 10 & 61 & M & Cecum & 11 & $26 / 22$ & LST-G & Category 3 & Yes/Yes & None \\
\hline
\end{tabular}

erative perforation are major advantages of hybrid ESD. These results indicate that hybrid ESD can be presented as an alternative or rescue method for colorectal ESD.

Although hybrid ESD seems to be a safe therapeutic method for the treatment of large colorectal tumors, the risk of delayed bleeding must be considered. In the present study, delayed bleeding occurred in 1 patient 24 hours after the hybrid ESD procedure; however, the complication was managed by endoscopic hemostasis and blood transfusions were not required. The patient was discharged from hospital 2 days after the hybrid ESD procedure. Compared with EMR, the delayed bleeding rate is reportedly low for ESD [7], probably because the small vessels are coagulated during the submucosal dissection. Careful observation of the post-procedural ulcer floor and prophylactic closure and/or coagulation of the submucosal blood vessels may help to reduce the risk of delayed bleeding after hybrid ESD.

Although a cutting device and additional snare are usually required for hybrid ESD [8], we demonstrated that all the hybrid ESD steps could be completed using the "SOUTEN" snare. Since the cost of ESD devices is 2 to 4 times higher than that of an EMR snare, cost effectiveness is a notable advantage of hybrid ESD using a "SOUTEN" snare. Additionally, this newly developed, multifunctional snare seems suitable for hybrid ESD for the following reasons. (1) The knob-shaped tip enables circumferential incision and submucosal dissection in all directions. (2) The length of the needle-knife can be locked, eliminating the need to maintain the cutting wire length during the procedure. (3) The loop is firm enough to prevent snare slippage. Together with its cost effectiveness, this device may help to promote the application of hybrid ESD in clinical practice.
This study had several limitations. Firstly, it may be difficult to apply this technique in cases with huge and/or severely fibrotic colorectal lesions. Although a proficient technique is required, conventional ESD may be a therapeutic option for such lesions [9]. Secondly, the overall number of patients included was comparatively small. Finally, our results were based on a patient series treated by 2 proficient endoscopists at a single center. Large, multicentered, prospective interventional studies, which are more likely to include ESD beginners, are therefore needed to standardize the use of hybrid ESD for the treatment of relatively large colorectal tumors.

In conclusion, hybrid ESD can be used to treat colorectal tumors that are $20-30 \mathrm{~mm}$ in diameter. The newly developed, multifunctional "SOUTEN" snare may help to achieve easy, safe, and cost-effective resections of such lesions.

\section{Competing interests}

None

References

[1] Tanaka S, Oka S, Kaneko I et al. Endoscopic submucosal dissection for colorectal neoplasia: possibility of standardization. Gastrointest Endosc 2007; 66: $100-107$

[2] Ohata K, Nonaka K, Misumi Y et al. Usefulness of training using animal models for colorectal endoscopic submucosal dissection: is experience performing gastric ESD really needed? Endosc Int Open 2016; 4: E333-E339 
[3] Toyonaga T, Man-I M, Morita Y et al. Endoscopic submucosal dissection (ESD) versus simplified/hybrid ESD. Gastrointest Endosc Clin N Am 2014; 24: 191 - 199

[4] Schlemper RJ, Riddell RH, Kato Y et al. The Vienna classification of gastrointestinal epithelial neoplasia. Gut 2000; 47: $251-255$

[5] Saito Y, Fukuzawa M, Matsuda T et al. Clinical outcome of endoscopic submucosal dissection versus endoscopic mucosal resection of large colorectal tumors as determined by curative resection. Surg Endosc 2010; $24: 343-352$

[6] Tanaka S, Haruma K, Oka S et al. Clinicopathologic features and endoscopic treatment of superficially spreading colorectal neoplasms larger than 20 mm. Gastrointest Endosc 2001; 54: 62-66
[7] Bae JH, Yang DH, Lee S et al. Optimized hybrid endoscopic submucosal dissection for colorectal tumors: a randomized controlled trial. Gastrointest Endosc 2016; 83: 584 - 592

[8] Fujiya M, Tanaka K, Dokoshi T et al. Efficacy and adverse events of EMR and endoscopic submucosal dissection for the treatment of coIon neoplasms: a meta-analysis of studies comparing EMR and endoscopic submucosal dissection. Gastrointest Endosc 2015; 81: 583 595

[9] Ohata K, Nonaka K, Minato Y et al. Endoscopic submucosal dissection for large colorectal tumor in a Japanese general hospital. J Oncol 2013; 2013: 218670 